Journal Afrika Statistika

Vol. 7, 2012, pages 474-490.

DOI: http://dx.doi.org/10.4314/afst.v7i1.7

Journal Afrika Statistika

\title{
ISSN 0852-0305 \\ Bias-corrected estimation in distortion risk premiums for heavy-tailed losses
}

\author{
Brahim Brahimi, Fatima Meddi and Abdelhakim Necir \\ Laboratory of Applied Mathematics, Mohamed Khider University of Biskra 07000, Algeria
}

Received 1 October 2011; Accepted 19 November 2012

Copyright (c) 2012, Journal Afrika Statistika. All rights reserved

\begin{abstract}
Recently Necir and Meraghni (2009) proposed an asymptotically normal estimator for distortion risk premiums when losses follow heavy-tailed distributions. In this paper, we propose a bias-corrected estimator of this class of risk premiums and establish its asymptotic normality. Our considerations are based on the high quantile estimator given by Matthys and Beirlant (2003).

Résumé. Récemment Necir and Meraghni (2009) ont proposé un estimateur asymptotiquement normal pour les primes de risque de distorsion lorsque les pertes suivent des distributions à queues lourdes. Dans cet article, nous proposons un estimateur à biais réduit, pour cette classe de primes de risque et nous établissons sa normalité asymptotique. Nos considérations sont basées sur l'estimateur des quantile extrêmes intoduit par Matthys and Beirlant (2003).
\end{abstract}

Key words: Bias reduction; High quantiles; Hill estimator; L-statistics; Order statistics; Risk Measure; Second order regular variation, Tail index.

AMS 2010 Mathematics Subject Classification : 91B30; 62G32; 62G30; 62 G05.

\section{Introduction}

Many premium calculation principles are introduced in actuarial science literature (e.g., Denuit et al. (1985); Furman and Zitikis (2008a) and references therein). The most popular ones are the net premium, the variance and standard deviation based premiums, the valueat-risk, the conditional tail expectation, and the proportional-hazards transform. Many of the premiums are special cases of the distortion premium (Wang, 1996), which is defined,

Brahim Brahimi : brah.brahim@gmail.com

Fatima Meddi: meddifatima@yahoo.fr

Abdelhakim Necir: necirabdelhakim@yahoo.fr 
for a given loss variable $X \geq 0$ with cumulative distribution function (cdf) $F$, by

$$
\Psi[F, g]=\int_{0}^{\infty} g(1-F(x)) d x
$$

where $g:[0,1] \rightarrow[0,1]$ is a distortion function, that is : $g$ is non-decreasing and such that $g(0)=0$ and $g(1)=1$. In term of quantile function $Q(s)=\inf \{x: F(x) \geq s\}, 0<s<1$, this class of risk premiums takes the following form

$$
\Psi[Q ; g]=\int_{0}^{1} Q(1-s) d g(s) .
$$

Most of the distortion functions $g$ are concave, which makes the corresponding distortion premiums $\Psi[F, g]$ coherent (Artzner et al., 1999 and Wirch and Hardy, 1999). In this paper, we are motivated by the risk losses for heavy-tailed distribution. Then it is quite natural to suppose that the distortion functions $g$ is such that $t \rightarrow g(1 / t)$ is regularly varying at infinity with index of regular variation $\rho \geq 1$, that is

$$
g(1 / t)=t^{-1 / \rho} \ell_{g}(t)
$$

where is $t \rightarrow \ell_{g}(t)$ is slowly varying as infinity, that is $\ell_{g}(t x) / \ell_{g}(t) \rightarrow 1$ as $t \rightarrow \infty$, for any $x>0$. Some examples of usual distortion functions satisfying regular variation condition (2) are listed below:

- Net premium

$$
g(x)=x \text { with } \rho=1, \ell_{g}(x)=1
$$

- Tail value-at-risk

$$
g(x)=\min \left(\frac{x}{p}, 1\right) \text { with } \rho=1, \ell_{g}(x)=1 ;
$$

- Proportional hazard transform (PHT)

$$
g(x)=x^{1 / \beta} \text { with } \rho=\beta, \ell_{g}(x)=1 ;
$$

- Dual power function principle $(0<\alpha<1)$

$$
g(x)=1-(1-x)^{\alpha} \text { with } \rho=1 / \alpha, \ell_{g}(x)=x^{-\alpha}-\left(x^{-1}-1\right)^{\alpha} ;
$$

- Gini principle $(0<\alpha \leq 1)$

$$
g(x)=(1+\alpha) x-\alpha x^{2} \text { with } \rho=1, \ell_{g}(x)=(1+\alpha)-\alpha x ;
$$

- Beta-distortion risk premium (Wirch and Hardy, 1999)

$$
g(x)=\int_{0}^{x} \frac{t^{a}(1-t)^{b}}{\beta(a, b)} d t \text { with } \rho=1 /(a+1), \ell_{g}(x)=\frac{1}{(a+1) \beta(a, b)}
$$

where $\beta(a, b):=\int_{0}^{1} \frac{t^{a}(1-t)^{b}}{\beta(a, b)} d t$. 
- MINMAXVAR2 risk premium (Madan and Schoutens, 2010)

$$
\begin{aligned}
& g(x)=1-\left(1-x^{\frac{1}{1+\lambda}}\right)^{1+\tau}, \lambda>0, \tau>0 \\
& \text { with } \rho=\frac{1+\lambda}{1+\tau}, \ell_{g}(x)=x^{-\frac{1+\tau}{1+\lambda}}-\left(x^{-\frac{1}{1+\lambda}}-1\right)^{1+\tau} .
\end{aligned}
$$

Jones and Zitikis (2003) noticed that the empirical counterpart of $\Psi(F ; g)$ is a linear combination of order statistics, commonly known as $L$-statistic. This opens up a fruitful venue for developing statistical inferential results, which have been actively investigated by a number of researchers. Specifically, let $X_{1}, \ldots, X_{n}$ be independent copies of $X$, and let $X_{1: n} \leq \cdots \leq X_{n: n}$ be the corresponding order statistics. The empirical estimator of the risk premium $\Psi[Q, g]$ is obtained by substituting $Q$ on the right-hand side of equation (1) by by its empirical counterpart $Q_{n}(s):=\inf \left\{x \in \mathbb{R}: F_{n}(x) \geq s\right\}, 0<s \leq 1$, associated to the empirical cdf defined on the real line, defined by $F_{n}(x):=n^{-1} \sum_{i=1}^{n} \mathbb{I}\left(X_{i} \leq x\right)$, with $\mathbb{I}(\cdot)$ being the indicator function. After straightforward computation, we obtain the formula

$$
\Psi\left[Q_{n} ; g\right]=\int_{0}^{1} Q_{n}(1-s) d g(s)=\sum_{i=1}^{n} a_{i, n}(g) X_{n-i+1: n},
$$

whose right-hand side is an $L$-statistic with the coefficients

$$
a_{i, n}(g):=g\left(\frac{i}{n}\right)-g\left(\frac{i-1}{n}\right) .
$$

For recent literature on statistical inference for distortion premiums, we refer to Jones and Zitikis (2003), Jones and Zitikis (2007), Centeno and Andrade (2005), Furman and Zitikis (2008a, 2008b), Brazauskas et al. (2008), Greselin et al. (2009), Necir and Meraghni (2009), Necir and Meraghni (2010), Brahimi et al. (2011), Peng et al. (2012) and the references therein.

Throughout this paper, the standard notation $\stackrel{p}{\rightarrow}, \stackrel{d}{\rightarrow}$ and $\stackrel{d}{=}$ respectively stand for convergence in probability, convergence in distribution and equality in distribution. For further notation we write $W_{n}^{(1)} \approx W_{n}^{(2)}$ to say that the two sequences of rv's $W_{n}^{(1)}, W_{n}^{(2)}$ are such that $W_{n}^{(1)} / W_{n}^{(2)} \stackrel{p}{\rightarrow} 1$. The symbol $\mathcal{N}\left(a, b^{2}\right)$ denotes the normal distribution with mean $a$ and variance $b^{2}$.

Jones and Zitikis (2003) employ asymptotic theory for L-statistics to prove that, for underlying distributions with a sufficient number of finite moments and under certain regularity conditions on the distortion function $g$, the empirical estimator of a risk premium $\Psi\left[F_{n} ; g\right]$ of $\Psi(F ; g)$ is strongly consistent and asymptotically normal. More precisely

$$
n^{1 / 2}\left(\Psi\left[F_{n} ; g\right]-\Psi[F ; g]\right) \stackrel{d}{\rightarrow} \mathcal{N}\left(0, \sigma_{F}^{2}\right), \text { as } n \rightarrow \infty,
$$

provided that the asymptotic variance

$$
\sigma_{F}^{2}:=\int_{0}^{1} \int_{0}^{1}(\min (s, t)-s t) g^{\prime}(s) g^{\prime}(t) d Q(1-s) d Q(1-t)
$$


is finite. The same authors are also discussed the PHT premiums estimators which corresponds to power distortion function $g(x)=x^{1 / \rho}, \rho \geq 1$. In this case the asymptotic normality (4) holds for any $1<\rho<2$, provided that

$$
\mathbf{E}\left(X^{\nu}\right)<\infty \text { for some } \nu>2 \rho /(2-\rho) .
$$

Empirical studies have shown that financial and actuarial data exhibit heavy tails or Pareto like distributions. The class of regularly varying cdf's is a major subclass of heavy-tailed distributions, it includes distributions such as Pareto, Burr, Student, Lévy-stable, and loggamma, which are known to be appropriate models for fitting large insurance claims, large fluctuations of prices, log-returns, etc. (see, e.g., Beirlant et al., 2001; Reiss and Thomas, 2007 and Rolski et al., 1999). The tail distribution of almost of these models may be written as follows

$$
1-F(x)=x^{-1 / \gamma} \ell_{F}(x), \text { for every } x>0,
$$

where $\gamma>0$ and $\boldsymbol{\ell}_{F}$ is a slowly varying function.

Suppose that $1-F(x)=x^{-1 / \gamma}, x \geq 1$. Then the risk premium PHT, which is greater than or equal to the mean risk, must be finite for any $\rho \geq 1$. This means that we have: $1 \leq \rho<1 / \gamma$. On the other hand, for $\gamma>1 / 2$, we have $1 \leq \rho<2$. However the second-order moment $\mathbf{E}\left(X^{2}\right)$ is infinite. In this case $2 \rho /(2-\rho)>2$, which implies that $\mathbf{E}\left(X^{\nu}\right)$ is infinite for any $\nu>2 \rho /(2-\rho)$. Hence, the range $\gamma \in(1 / 2,1)$ is not covered by the central limit theorem (4) and thus we need to seek another approach to handle this situation.

By making use of the extreme value theory (see for instance de Haan and Ferreira, 2006), Necir and Meraghni (2009) proposed an alternative estimator for $\Psi[F, g]$ and established its asymptotic normality for any $\gamma \in(1 / 2,1)$. Though this estimator enjoys the asymptotic normality property, it still has a problem due to the fact that, it is based on Weissman's estimator of high quantiles known to be largely biased. Several estimators with reduced biases are proposed in the literature as alternatives to Weissman's ones, see for instance, Feureverger and Hall (1999), Beirlant et al. (2002), Gomes and Martins (2002), Gomes and Martins (2004), Caeiro et al. (2004), Caeiro et al. (2009), Peng and Qi (2004), Matthys and Beirlant (2003), Gomes and Figueiredo (2006) and Gomes and Pestana (2004). Actually, we are interested in bias-reduced estimators of high quantiles with nice asymptotic properties that provide us Gaussian approximations leading to confidence bounds for $\Psi[F, g]$. Our choice is the one proposed by Matthys and Beirlant (2003).

The rest of the paper is organized as follows. In Section 2, we present a short introduction on the risk premium estimator in the case of heavy-tailed losses which proposed by Necir and Meraghni (2009) and then define a new reduced-biased estimator of this class of risk premiums. We state our main results in Section 3 whose proofs are postponed to Section 4. Some notes and remarks are made in Section 5.

\section{Heavy-tailed losses case}

Let us define the following estimator for $Q$ :

$$
\widehat{Q}_{n}(1-s):= \begin{cases}Q_{n}^{W}(1-s) & \text { for } 0<s<k / n \\ Q_{n}(1-s) & \text { for } k / n \leq s<1,\end{cases}
$$


where $Q_{n}^{W}(1-s):=(k / n)^{\widehat{\gamma}_{n}^{H}} X_{n-k: n} s^{-\widehat{\gamma}_{n}^{H}}, s \downarrow 0$, is Weissman's estimator (Weissman, 1978) of high quantiles, with

$$
\widehat{\gamma}_{n}^{H}:=k^{-1} \sum_{i=1}^{k} \log \left(\frac{X_{n-i+1: n}}{X_{n-k: n}}\right)
$$

being the well-known Hill estimator (Hill, 1975) of the tail index $\gamma$. The integer sequence $k=k_{n}$, represents the number of upper order statistics used in the computation of $\widehat{\gamma}_{n}^{H}$, satisfying

$$
1<k<n, k \rightarrow \infty \text { and } k / n \rightarrow 0 \text { as } n \rightarrow \infty .
$$

By replacing $Q(1-s)$ by $\widehat{Q}_{n}(1-t)$ in formula (1), Necir and Meraghni (2009) proposed an alternative estimator for $\Psi[Q ; g]$ as follows:

$$
\Psi\left[\widehat{Q}_{n} ; g\right]:=\int_{0}^{1} \widehat{Q}_{n}(1-s) d g(s)=\int_{0}^{k / n} Q_{n}^{W}(1-s) d g(s)+\int_{k / n}^{1} Q_{n}(1-s) d g(s)
$$

which, by a straightforward calculation, is equal to

$$
\Psi\left[\widehat{Q}_{n} ; g\right]:=g(k / n) \frac{X_{n-k: n}}{1-\rho \widehat{\gamma}_{n}^{H}}+\sum_{i=k+1}^{n} a_{i, n}(g) X_{n-i+1: n}
$$

where $a_{i, n}(g)$ is that defined in (3) provided that $\rho \widehat{\gamma}_{n}^{H} \in(0,1)$. Moreover, the same author showed that, under suitable regularity assumptions, for any $\gamma \in(1 / 2,1)$,

$$
\frac{\sqrt{n}\left(\Psi\left[\widehat{Q}_{n} ; g\right]-\Psi[Q ; g]\right)}{(k / n)^{-1 / 2} g(k / n) Q(1-k / n)} \stackrel{d}{\rightarrow} \mathcal{N}\left(0, \sigma^{2}(\gamma ; \rho)\right), \text { as } n \rightarrow \infty
$$

where

$$
\sigma^{2}(\rho, \gamma)=\frac{\gamma^{2}\left(\gamma^{2} \rho^{2}-2 \gamma^{2} \rho^{3}+4 \gamma \rho^{2}-2 \gamma \rho+\rho^{2}-2 \rho+1\right)}{(\gamma \rho-1)^{4}}+\frac{2 \gamma^{2}(\rho+\gamma \rho-1)}{(\gamma \rho-1)^{2}(\rho+2 \gamma \rho-2)} .
$$

Matthys-Beirlant's estimator of high quantiles is defined as follows

$$
Q_{n}^{\mathrm{MB}}(1-s)=X_{n-k: n}+\widehat{a}_{n, k} \frac{(k / n)^{\widehat{\gamma}_{k}} s^{-\widehat{\gamma}_{k}}-1}{\widehat{\gamma}_{k}}, 0<s<k / n,
$$

where $\widehat{\gamma}_{k}$ is a maximum likelihood estimator of tail index $\gamma>0$, defined by

$$
\widehat{\gamma}_{k}=\arg \max _{\gamma>0} \sum_{i=1}^{k-1}\left\{\log \frac{1-\left(\frac{j}{k+1}\right)^{\gamma}}{\gamma}-\frac{1-\left(\frac{j}{k+1}\right)^{\gamma}}{\gamma} Y_{j}\right\},
$$

and

$$
\widehat{a}_{n, k}=\frac{1}{k} \sum_{i=1}^{k} j\left(X_{n-j+1: n}-X_{n-j: n}\right)\left(\frac{j}{k+1}\right)^{\widehat{\gamma}_{k}}
$$

with

$$
Y_{j}:=j \log \frac{X_{n-j+1: n}-X_{n-k: n}}{X_{n-j: n}-X_{n-k: n}}, 1 \leq j<k .
$$


Instead of $\widehat{Q}_{n}$, let us define the following estimator for $Q$ :

$$
\widetilde{Q}_{n}(1-s):= \begin{cases}Q_{n}^{\mathrm{MB}}(1-s) & \text { for } \quad 0<s<\frac{k}{n} \\ Q_{n}(1-s) & \text { for } \frac{k}{n} \leq s<1,\end{cases}
$$

Then the bias-reduced estimator of $\Psi[Q ; g]$ is

$$
\Psi\left[\widetilde{Q}_{n} ; g\right]=\int_{0}^{1} \widetilde{Q}_{n}(1-s) d g(s),
$$

which by straightforward computation yields

$$
\Psi\left[\widetilde{Q}_{n} ; g\right]=g(k / n) X_{n-k: n}+g(k / n) \frac{\rho \widehat{a}_{n, k}}{1-\rho \widehat{\gamma}_{k}}+\sum_{i=k+1}^{n} a_{i, n}(g) X_{n-i+1: n},
$$

where the coefficients $a_{i, n}(g)$ are as in (3), observe that $a_{n, k} \sim \gamma Q(1-k / n)$. Hence it is easy to show that $a_{n, k}=\widehat{\gamma}_{k} X_{n-k: n}+o_{p}(1)$. It is clear that the choice of $a_{n, k}=\widehat{\gamma}_{k} X_{n-k: n}$, in (7), coincides with that of Necir and Meraghni (2009).

\section{Main results}

Let us introduce now the tail quantile function defined by

$$
\mathbb{U}(t):=(1 /(1-F))^{-1}(t)=Q(1-1 / t), 1<t<\infty .
$$

Notice that the regular variation condition (5) implies

$$
\lim _{t \rightarrow \infty} \mathbb{U}(t x) / \mathbb{U}(t)=x^{\gamma}, \text { for any } x>0 .
$$

The regular condition itself is not sufficient for establishing asymptotic distributions. To this end, we strengthen the condition (8), into the following one: the cdf $F$ is said to fulfill the second-order regular-variation with second-order parameter $\eta \leq 0$ if there exists a function $t \rightarrow A(t)$, converging to zero when $t$ tends to infinity, has a constant sign for large values of $t$, and such that

$$
\lim _{t \rightarrow \infty} \frac{\frac{\mathbb{U}(t x)}{\mathbb{U}(t)}-\frac{x^{\gamma}-1}{\gamma}}{A(t)}=\int_{1}^{x} s^{\gamma-1} \int_{1}^{s} v^{\eta-1} d v d s,
$$

For more details, see Section 2.3 page 43 in (de Haan and Ferreira, 2006).

Theorem 1. Assume that the second order condition (9) holds with the function $A(\cdot)$ and let $k=k_{n}$ be an integer sequence satisfying (6) with $\sqrt{k} A(n / k) \rightarrow 0$ as $n \rightarrow \infty$. Then there exists a sequence of Brownian bridges $\left\{B_{n}(t), 0 \leq t \leq 1\right\}$ such that for all large $n$

$$
a_{\gamma} \sqrt{k}\left(\widehat{\gamma}_{k}-\gamma\right) \approx \sqrt{\frac{n}{k}} B_{n}\left(\frac{k}{n}\right) \int_{0}^{1} t d K_{\gamma}^{(1)}(t)-\sqrt{\frac{n}{k}} \int_{0}^{1} B_{n}\left(\frac{k}{n} t\right) d K_{\gamma}^{(1)}(t),
$$

and, for a sequence $a_{n, k}=\gamma \mathbb{U}(n / k)$, we have

$$
\sqrt{k}\left(\frac{\widehat{a}_{n, k}}{a_{n, k}}-1\right) \approx \sqrt{\frac{n}{k}} B_{n}\left(\frac{k}{n}\right) \int_{0}^{1} t d K_{\gamma}^{(2)}(t)-\sqrt{\frac{n}{k}} \int_{0}^{1} B_{n}\left(\frac{k}{n} t\right) d K_{\gamma}^{(2)}(t)+\gamma \sqrt{\frac{n}{k}} B_{n}\left(\frac{k}{n}\right),
$$


where

$$
\begin{gathered}
K_{\gamma}^{(1)}(t):=\frac{1}{\gamma} \log t+\frac{1+\gamma}{\gamma^{2}} \int_{1}^{t^{\gamma}} \frac{\log u}{1-u} d u, \quad(t \geq 0), \\
K_{\gamma}^{(2)}(t):=(\gamma+1) \log t+a_{\gamma}^{-1} K_{\gamma}^{(1)}(t),
\end{gathered}
$$

and

$$
a_{\gamma}:=\gamma^{-2} \int_{0}^{1}\left(1-u^{\gamma}+u^{\gamma} \log u^{\gamma}\right)^{2}\left(1-u^{\gamma}\right)^{-2} d u
$$

Corollary 1. Under the assumptions of Theorem 1, we have

$$
\left(\sqrt{k}\left(\widehat{\gamma}_{k}-\gamma\right), \sqrt{k}\left(\frac{\widehat{a}_{n, k}}{a_{n, k}}-1\right)\right) \stackrel{d}{\rightarrow} \mathcal{N}\left(0, \sum_{\gamma}^{2}\right)
$$

where $\sum_{\gamma}^{2}:=\left(\sigma_{i j}^{2}\right)_{i, j=1,2}$ is the asymptotic variance-covariance matrix defined by

$$
\sigma_{i j}^{2}=\sigma_{j i}^{2}=\operatorname{Cov}\left[K_{\gamma}^{(i)}(\xi), K_{\gamma}^{(j)}(\xi)\right], i \neq j,
$$

$\sigma_{1}^{2}=\operatorname{Var}\left[K_{\gamma}^{(1)}(\xi)\right]$ and $\sigma_{2}^{2}=\operatorname{Var}\left[K_{\gamma}^{(2)}(\xi)\right]+\gamma^{2}$, with $\xi$ is an $(0,1)$-uniform $r v$.

Theorem 2. Assume that the second-order regular-variation condition (9) holds with some $\gamma \in(1 / 2,1)$, and let the quantile function $Q$ be continuous on the interval $(0,1)$. Furthermore, let $k=k_{n}$ be integers satisfying condition (6) such that $\sqrt{k} A(n / k) \rightarrow 0$ when $n \rightarrow \infty$. Then, for any $1<\rho<1 / \gamma$, we have

$$
\begin{aligned}
\frac{\sqrt{n}\left(\Psi\left[\widetilde{Q}_{n} ; g\right]-\Psi[Q ; g]\right)}{(k / n)^{-1 / 2} g(k / n) a_{n, k}} & =\sqrt{\frac{n}{k}} B_{n}\left(\frac{k}{n}\right) \int_{0}^{1} t d K_{\gamma}^{(3)}(t)-\sqrt{\frac{n}{k}} \int_{0}^{1} B_{n}\left(\frac{k}{n} t\right) d K_{\gamma}^{(3)}(t) \\
& +\frac{1}{1-\rho \gamma} \sqrt{\frac{n}{k}} B_{n}\left(\frac{k}{n}\right)-\frac{\int_{0}^{1-k / n} g^{\prime}(1-t) B_{n}(t) d Q(t)}{(k / n)^{-1 / 2} g(k / n) a_{n, k}}
\end{aligned}
$$

where

$$
K_{\gamma}^{(3)}(t)=\frac{\rho^{2}}{(1-\rho \gamma)^{2}} K_{\gamma}^{(1)}(t)+\frac{\rho}{1-\rho \gamma} K_{\gamma}^{(2)}(t) .
$$

Corollary 2. Under the assumptions of Theorem 2, we have

$$
\frac{\sqrt{n}\left(\Psi\left[\widetilde{Q}_{n} ; g\right]-\Psi[Q ; g]\right)}{(k / n)^{-1 / 2} g(k / n) a_{n, k}} \stackrel{d}{\rightarrow} \mathcal{N}\left(0, D^{2}(\gamma, \rho)\right), \text { as } n \rightarrow \infty
$$

where

$D^{2}(\gamma, \rho)=\operatorname{Var}\left[K_{\gamma}^{(3)}(\xi)\right]+\frac{1}{(1-\rho \gamma)^{2}}+\frac{2 \rho^{2}}{1-\rho-\gamma \rho}\left(\frac{\rho+3 \gamma \rho-3}{(1-\gamma \rho)(\rho+2 \gamma \rho-2)}+\int_{0}^{1} t d K_{\gamma}^{(3)}(t)\right)$ 


\section{Proofs}

\subsection{Proof of Theorem 1}

4.1.1. Asymptotic distribution of $\widehat{\gamma}_{k}$

First, for convenience, we set

$$
\mathcal{L}(\gamma ; \mathbf{Y}):=\sum_{i=1}^{k-1}\left\{\log \frac{1-\left(\frac{j}{k+1}\right)^{\gamma}}{\gamma}-\frac{1-\left(\frac{j}{k+1}\right)^{\gamma}}{\gamma} Y_{j}\right\},
$$

with $\mathbf{Y}:=\left(Y_{1}, \ldots, Y_{k-1}\right)$. By construction, the tail index estimator $\widehat{\gamma}_{k}$ of $\gamma$, proposed by Matthys and Beirlant (2003), is the value of $\gamma$ that maximizes the quantity $\mathcal{L}(\gamma ; \mathbf{Y})$. In other terms, $\widehat{\gamma}_{k}$ is the solution of the following equation

$$
\frac{\partial \mathcal{L}(\gamma ; \mathbf{Y})}{\partial \gamma}=0
$$

Making use of a Taylor expansion to function $\gamma \rightarrow \frac{\partial \mathcal{L}(\gamma ; \mathbf{Y})}{\partial \gamma}$ around $\widehat{\gamma}_{k}$, we obtain

$$
\left[\frac{\partial \mathcal{L}(\gamma ; \mathbf{Y})}{\partial \gamma}\right]_{\gamma=\widehat{\gamma}_{k}}-\frac{\partial \mathcal{L}(\gamma ; \mathbf{Y})}{\partial \gamma}=\frac{1}{k}\left[\frac{\partial^{2} \mathcal{L}(\gamma ; \mathbf{Y})}{\partial^{2} \gamma}\right]_{\gamma=\widehat{\gamma}_{k}^{*}}\left(\widehat{\gamma}_{k}-\gamma\right)
$$

Since $\widehat{\gamma}_{k}$ is the solution of equation (11), then

$$
\left(\widehat{\gamma}_{k+1}-\gamma\right) \frac{1}{k}\left[\frac{\partial^{2} \mathcal{L}(\gamma ; \mathbf{Y})}{\partial^{2} \gamma}\right]_{\gamma=\widehat{\gamma}_{k}^{*}}=-\frac{\partial \mathcal{L}(\gamma ; \mathbf{Y})}{\partial \gamma}
$$

On the other hand, Matthys and Beirlant (2003) showed that, under the second order condition (9),

$$
\frac{1}{\sqrt{k}} \frac{\partial^{2} \mathcal{L}(\gamma ; \mathbf{Y})}{\partial^{2} \gamma}=-a_{\gamma}+o_{p}(1), \text { as } n \rightarrow \infty
$$

where $a_{\gamma}$ is the one defined in (10). Therefore, we have

$$
a_{\gamma} \sqrt{k}\left(\widehat{\gamma}_{k}-\gamma\right) \approx \frac{1}{\sqrt{k}} \frac{\partial \mathcal{L}(\gamma ; \mathbf{Y})}{\partial \gamma}
$$

Our task now is to approximate, asymptotically, the quantity $\frac{1}{\sqrt{k}} \frac{\partial \mathcal{L}(\gamma ; \mathbf{Y})}{\partial \gamma}$ by a sequence of Gaussian rv's. More precisely, we will represent this quantity by the uniform quantile processes, defined below, and then make use of the weak approximation given in Theorem 2.1 of Csörgö et al. (1986). To this end, let $\xi_{1}, \xi_{2}, \ldots$ be independent uniform $(0,1)$ rv's and for each $n \geq 1$, and define

$$
\mathbb{G}_{n}(t):=\frac{1}{n} \sum_{i=1}^{n} \mathbb{I}\left(\xi_{i} \leq t\right), 0 \leq t \leq 1,
$$

Journal home page: www.jafristat.net 
the uniform empirical distribution function, defined to be right continuous, based on $\xi_{1}, \ldots, \xi_{n}$ via indicator function $\mathbb{I}(\cdot)$. The uniform empirical quantile function is given by

$$
\mathbb{V}_{n}(t):=\inf \left\{s: \mathbb{G}_{n}(s) \geq t\right\}, 0 \leq s \leq 1, \mathbb{V}_{n}(0):=\mathbb{V}_{n}(0+),
$$

that is $\mathbb{V}_{n}$ is defined to be the left continuous inverse of the empirical distribution function $\mathbb{G}_{n}$. In term of the order statistic $\xi_{1: n} \leq \ldots \leq \xi_{n: n}$, pertaining to the sample $\xi_{1}, \ldots, \xi_{n}$, we have $\mathbb{V}_{n}(t)=\xi_{i: n},(i-1) / n<t \leq i / n$. The corresponding uniform quantile process is defined by

$$
\beta_{n}(t):=\sqrt{n}\left(t-\mathbb{V}_{n}(t)\right), 0 \leq t \leq 1 .
$$

The two sequences of order statistics $X_{1: n} \leq \ldots \leq X_{n: n}$ and $\xi_{1: n} \leq \ldots \leq \xi_{n: n}$ are linked via the following equality in distribution

$$
\left\{X_{n-j+1: n}\right\}_{j=1}^{n} \stackrel{d}{=}\left\{\mathbb{U}\left(\xi_{j: n}^{-1}\right)\right\}_{j=1}^{n} .
$$

Matthys and Beirlant (2003) also showed that for all large $n$

$$
\frac{1}{\sqrt{k}} \frac{\partial \mathcal{L}(\gamma ; \mathbf{Y})}{\partial \gamma}=\frac{1}{\sqrt{k}} \sum_{j=2}^{k-1} c_{k, j} H\left(\frac{\xi_{j: n}}{\xi_{k+1: n}}\right)-\sqrt{k} \mu_{k}+o_{p}(1)
$$

where $H(t)=-\log \left(t^{-\gamma}-1\right)$,

$$
c_{k, j}=-(k+1) \int_{\frac{j-1}{k+1}}^{\frac{j}{k+1}} J(s) d s \text { and } \mu_{k}=\sum_{j=2}^{k-1} c_{k, j} \int_{\frac{1}{k+1}}^{\frac{k}{k+1}} H(t) d t .
$$

It is easy to show that $\mu_{k}=\int_{\frac{1}{k+1}}^{\frac{k}{k+1}} J(t) H(t) d t+O\left(k^{-1}\right)$. So it follows that

$$
\frac{1}{\sqrt{k}} \frac{\partial \mathcal{L}(\gamma ; \mathbf{Y})}{\partial \gamma}=\frac{1}{\sqrt{k}} \sum_{j=2}^{k-1} c_{k, j} H\left(\frac{\xi_{j: n}}{\xi_{k+1: n}}\right)-\sqrt{k} \int_{\frac{1}{k+1}}^{\frac{k}{k+1}} J(t) H(t) d t+o_{p}(1)
$$

In the sequel, for sake of conciseness, to avoid large formulas, we set $\omega_{n, k}(t)=$ $\frac{\mathbb{V}_{n}\left(\frac{k+1}{n} t\right)}{\xi_{k+1: n}}$. Now, we make use of the empirical quantile function $\mathbb{V}_{n}(\cdot)$ and rewrite the first term of the right hand of the previous equation into

$$
\frac{k+1}{\sqrt{k}} \int_{\frac{1}{k+1}}^{\frac{k}{k+1}} J(t) H\left(\omega_{n, k}(t)\right) d t .
$$

By using the mean value theorem to function $t \rightarrow H(t)$, we get

$$
H\left(\omega_{n, k}(t)\right)-H(t)=H^{\prime}\left(\vartheta_{n, k}(t)\right)\left(\omega_{n, k}(t)-t\right),
$$

where $\vartheta_{n, k}(t)$ is a sequence of rv's belongs to the open interval with endpoints $\omega_{n, k}(t)$ and $t$. Thus we arrive at

$$
\frac{1}{\sqrt{k}} \frac{\partial \mathcal{L}(\gamma ; \mathbf{Y})}{\partial \gamma} \approx \frac{k+1}{\sqrt{k}} \int_{\frac{1}{k+1}}^{\frac{k}{k+1}} J(t) H^{\prime}(t)\left(\omega_{n, k}(t)-t\right) d t+R_{n}^{(1)}
$$


where

$$
R_{n}^{(1)}:=\frac{k+1}{\sqrt{k}} \int_{\frac{1}{k+1}}^{\frac{k}{k+1}} J(t)\left(\frac{H^{\prime}\left(\vartheta_{n, k}(t)\right)}{H^{\prime}(t)}-1\right) H^{\prime}(t)\left(\omega_{n, k}(t)-t\right) d t+o_{p}(1) .
$$

We may and do treat this remainder term $R_{n}^{(1)}$ as did Necir and Meraghni (2009) in their Theorem 1 and show that it tends to zero in probability as $n \rightarrow \infty$ by omitting the details. From Smirnov's Lemma (see Lemma 2.2.3 in de Haan and Ferreira, 2006, page 41) we have $\xi_{k+1: n} \approx k / n$, it follows that the first term of the right-hand of the equation (13) is

$$
\approx \frac{n}{\sqrt{k}} \int_{\frac{1}{k+1}}^{\frac{k}{k+1}} J(t)\left(\mathbb{V}_{n}\left(\frac{k+1}{n} t\right)-\xi_{k+1: n} t\right) d H(t) .
$$

Observe now that the previous expression is equal to

$$
\begin{aligned}
\frac{n}{\sqrt{k}} \int_{\frac{1}{k+1}}^{\frac{k}{k+1}} J(t)( & \left.\mathbb{V}_{n}\left(\frac{k+1}{n} t\right)-\frac{k+1}{n} t\right) d H(t) \\
& -\frac{n}{\sqrt{k}} \int_{\frac{1}{k+1}}^{\frac{k}{k+1}} J(t)\left(\xi_{k+1: n} t-\frac{k+1}{n} t\right) d H(t) .
\end{aligned}
$$

Making use of the uniform empirical quantile process $\beta_{n}(\cdot)$, defined in (12), we write

$$
\begin{aligned}
\frac{1}{\sqrt{k}} \frac{\partial \mathcal{L}(\gamma ; \mathbf{Y})}{\partial \gamma} \approx-\sqrt{\frac{n}{k}} \int_{\frac{1}{k+1}}^{\frac{k}{k+1}} J(t) \beta_{n}\left(\frac{k+1}{n} t\right) d H(t) \\
+\sqrt{\frac{n}{k}} \beta_{n}\left(\frac{k+1}{n}\right) \int_{\frac{1}{k+1}}^{\frac{k}{k+1}} t J(t) d H(t) .
\end{aligned}
$$

From Theorem 2.1 of Csörgő et al. (1986), on a suitable probability space, there exists a sequence of Brownian bridges $\left\{B_{n}(t), 0 \leq t \leq 1\right\}$ such that, for every $0 \leq \delta<1 / 2$, we have, as $n \rightarrow \infty$

$$
\sup _{1 / n \leq s \leq 1-1 / n} \frac{\left|\beta_{n}(s)-B_{n}(s)\right|}{(s(1-s))^{1 / 2-\delta}}=O_{p}\left(n^{-\tau}\right) .
$$

By making use of this approximation, we readily get

$$
\begin{aligned}
\frac{1}{\sqrt{k}} \frac{\partial \mathcal{L}(\gamma ; \mathbf{Y})}{\partial \gamma} & =-\sqrt{\frac{n}{k}} \int_{\frac{1}{k+1}}^{\frac{k}{k+1}} J(t) B_{n}\left(\frac{k+1}{n} t\right) d H(t) \\
& +\sqrt{\frac{n}{k}} B_{n}\left(\frac{k+1}{n}\right) \int_{\frac{1}{k+1}}^{\frac{k}{k+1}} t J(t) d H(t)+R_{n}^{(2)}
\end{aligned}
$$

Here again, showing that the remainder term $R_{n}^{(2)}$ tends to zero in probability is straightforward and we the omit the proof. It easy to show that

$$
\sqrt{\frac{n}{k}} \int_{\frac{1}{k+1}}^{\frac{k}{k+1}} J(t) B_{n}\left(\frac{k+1}{n} t\right) d H(t) \approx \sqrt{\frac{n}{k}} \int_{0}^{1} J(t) B_{n}\left(\frac{k}{n} t\right) d H(t)
$$


and

$$
\sqrt{\frac{n}{k}} B_{n}\left(\frac{k+1}{n}\right) \int_{\frac{1}{k+1}}^{\frac{k}{k+1}} t J(t) d H(t) \approx \sqrt{\frac{n}{k}} B_{n}\left(\frac{k}{n}\right) \int_{0}^{1} t J(t) d H(t) .
$$

Finally we obtain

$$
\frac{1}{\sqrt{k}} \frac{\partial \mathcal{L}(\gamma ; \mathbf{Y})}{\partial \gamma} \approx \sqrt{\frac{n}{k}} B_{n}\left(\frac{k}{n}\right) \int_{0}^{1} t J(t) d H(t)-\sqrt{\frac{n}{k}} \int_{0}^{1} J(t) B_{n}\left(\frac{k}{n} t\right) d H(t) .
$$

By letting $d K_{\gamma}^{(1)}(t)=-J(t) d H(t)$, we get

$$
a_{\gamma} \frac{1}{\sqrt{k}} \frac{\partial \mathcal{L}(\gamma ; \mathbf{Y})}{\partial \gamma} \approx \sqrt{\frac{n}{k}} \int_{0}^{1} B_{n}\left(\frac{k}{n} t\right) d K_{\gamma}^{(1)}(t)-\sqrt{\frac{n}{k}} B_{n}\left(\frac{k}{n}\right) \int_{0}^{1} t d K_{\gamma}^{(1)}(t),
$$

this achives the proof of first part of Theorem 1 .

\subsection{Asymptotic distribution of $\widehat{a}_{n, k}$}

Recall that $a_{n, k}=\gamma \mathbb{U}(n / k)$. By letting $\widetilde{a}_{n, k}=\gamma \mathbb{U}\left(\xi_{k+1: n}^{-1}\right)$ we write

$$
\frac{\widehat{a}_{n, k}}{a_{n, k}}-1=\left(\frac{\widehat{a}_{n, k}}{\widetilde{a}_{n, k}}-1\right)+\frac{\widehat{a}_{n, k}}{\widetilde{a}_{n, k}}\left(\frac{\widetilde{a}_{n, k}}{a_{n, k}}-1\right) .
$$

Next we show that both of the two terms of (15) are asymptotically centred Gaussian rv's.

Making use of the second order condition (9), we get

$$
\frac{X_{n-j+1: n}-X_{n-j: n}}{a_{n, k}} \approx \frac{\left(\frac{\xi_{j: n}}{\xi_{k+1: n}}\right)^{-\gamma}-\left(\frac{\xi_{j+1: n}}{\xi_{k+1: n}}\right)^{-\gamma}}{\gamma}, \text { for } 1 \leq j \leq k .
$$

We also have

$$
\begin{aligned}
\frac{\widehat{a}_{n, k}}{a_{n, k}} & =\frac{1}{k} \sum_{j=1}^{k} j \frac{X_{n-j+1: n}-X_{n-j: n}}{a_{n, k}}\left(\frac{j}{k+1}\right)^{\widehat{\gamma}_{k}} \\
& \approx \frac{1}{k} \sum_{j=1}^{k} j \frac{\left(\frac{\xi_{j: n}}{\xi_{k+1: n}}\right)^{-\gamma}-\left(\frac{\xi_{j+1: n}}{\xi_{k+1: n}}\right)^{-\gamma}}{\gamma}\left(\frac{j}{k+1}\right)^{\widehat{\gamma}_{k}}=: \Delta_{n, k} .
\end{aligned}
$$

This last expression may be rewritten into

$$
\gamma^{-1} \frac{k+1}{k} \xi_{k+1, n}^{\gamma} \sum_{j=1}^{k}\left(\xi_{j: n}^{-\gamma}-\xi_{j+1: n}^{-\gamma}\right)\left(\frac{j}{k+1}\right)^{\widehat{\gamma}_{k}+1}
$$

It is easy to verify that

$$
\begin{aligned}
& \sum_{j=1}^{k}\left(\xi_{j ; n}^{-\gamma}-\xi_{j+1: n}^{-\gamma}\right)\left(\frac{j}{k+1}\right)^{\widehat{\gamma}_{k}+1} \\
& =\sum_{j=1}^{k}\left[\left(\frac{j}{k+1}\right)^{\widehat{\gamma}_{k}+1}-\left(\frac{j-1}{k+1}\right)^{\widehat{\gamma}_{k}+1}\right] \xi_{j, n}^{-\gamma}-\left(\frac{k}{k+1}\right)^{\widehat{\gamma}_{k}+1} \xi_{k+1, n}^{-\gamma} .
\end{aligned}
$$


Therefore

$$
\Delta_{n, k}=\gamma^{-1} \frac{k+1}{k} \xi_{k+1: n}^{\gamma} \sum_{j=1}^{k}\left[\left(\frac{j}{k+1}\right)^{\widehat{\gamma}_{k}+1}-\left(\frac{j-1}{k+1}\right)^{\widehat{\gamma}_{k}+1}\right] \xi_{j: n}^{-\gamma}-\gamma^{-1}\left(\frac{k}{k+1}\right)^{\widehat{\gamma}_{k}}
$$

which is equal to

$$
\gamma^{-1}\left(\widehat{\gamma}_{k}+1\right) \frac{k+1}{k}\left(\frac{n}{k+1}\right)^{\widehat{\gamma}_{k}+1} \xi_{k+1: n}^{\gamma} \sum_{j=1}^{k} \xi_{j: n}^{-\gamma} \int_{\frac{j-1}{n}}^{\frac{j}{n}} t^{\widehat{\gamma}} d t-\gamma^{-1}\left(\frac{k}{k+1}\right)^{\widehat{\gamma}_{k}} .
$$

Making use of the uniform empirical quantile function $\mathbb{V}_{n}(\cdot)$, we rewrite $\Delta_{n, k}$ as

$$
\gamma^{-1}\left(\widehat{\gamma}_{k}+1\right) \frac{k+1}{k}\left(\frac{n}{k+1}\right)^{\widehat{\gamma}_{k}+1} \xi_{k+1: n}^{\gamma} \int_{0}^{k / n} s^{\widehat{\gamma}_{k}} \mathbb{V}_{n}^{-\gamma}(s) d s-\gamma^{-1}\left(\frac{k}{k+1}\right)^{\widehat{\gamma}_{k}}
$$

which, by integration by parts, equals

$$
\gamma^{-1}\left(\widehat{\gamma}_{k}+1\right)\left(\frac{k}{k+1}\right)^{\widehat{\gamma}_{k}} \xi_{k+1: n}^{\gamma} \int_{0}^{1} s^{\widehat{\gamma}_{k}} \mathbb{V}_{n}^{-\gamma}(k s / n) d s-\gamma^{-1}\left(\frac{k}{k+1}\right)^{\widehat{\gamma}_{k}}
$$

Therefore

$$
\begin{aligned}
\Delta_{n, k} & =\gamma^{-1}\left(\frac{k}{k+1}\right)^{\widehat{\gamma}_{k}}\left[\left(\widehat{\gamma}_{k}+1\right) \xi_{k+1: n}^{\gamma} \int_{0}^{1} t^{\widehat{\gamma}_{k}} \mathbb{V}_{n}^{-\gamma}(k t / n) d t-1\right] . \\
& =: \gamma^{-1}\left(\frac{k}{k+1}\right)^{\widehat{\gamma}_{k}}\left(\Delta_{n, k}^{*}-1\right) .
\end{aligned}
$$

By the fact that $\left(\frac{k}{k+1}\right)^{\widehat{\gamma}_{k}}=1+O_{p}\left(k^{-1}\right)$, we infer that

$$
\left(\widehat{a}_{n, k} / a_{n, k}-1\right)=\left(1+O_{p}\left(k^{-1}\right)\right) \gamma^{-1}\left(\Delta_{n, k}^{*}-1\right)-1 .
$$

Hence

$$
\sqrt{k}\left(\frac{\widehat{a}_{n, k}}{a_{n, k}}-1\right)=\sqrt{k} \gamma^{-1}\left(\Delta_{n, k}^{*}-1-\gamma\right)+\sqrt{k} O_{p}\left(k^{-1}\right) .
$$

Since $\sqrt{k} O_{p}\left(k^{-1}\right) \rightarrow 0$ in probability, it suffices to show that $\sqrt{k}\left(\Delta_{n, k}^{*}-1-\gamma\right)$ is asymptotically centred Gaussian rv. Indeed, first notice that

$$
\Delta_{n, k}^{*}=\left(\widehat{\gamma}_{k}+1\right)\left(n \xi_{k+1: n} / k\right)^{\gamma}(k / n)^{\gamma} \int_{0}^{1} t^{\widehat{\gamma}_{k}} \mathbb{V}_{n}^{-\gamma}(k t / n) d t .
$$

It is east to verify that

$$
\Delta_{n, k}^{*}-1-\gamma=: S_{n} T_{n 1}+T_{n 2}+T_{n 3}+T_{n 4},
$$

where

$$
\begin{gathered}
S_{n}=\left(\widehat{\gamma}_{k}+1\right)\left(\frac{k}{n}\right)^{\gamma} \int_{0}^{1} t^{\widehat{\gamma}_{k}} \mathbb{V}_{n}^{-\gamma}\left(\frac{k}{n} t\right) d t \\
T_{n 1}=\left(n \xi_{k+1: n} / k\right)^{\gamma}-1
\end{gathered}
$$

Journal home page: www.jafristat.net 


$$
\begin{gathered}
T_{n 2}=\left(\widehat{\gamma}_{k}+1\right)\left(\frac{k}{n}\right)^{\gamma} \int_{0}^{1}\left(t^{\widehat{\gamma}_{k}}-t^{\gamma}\right) \mathbb{V}_{n}^{-\gamma}\left(\frac{k}{n} t\right) d t, \\
T_{n 3}=\left(\widehat{\gamma}_{k}+1\right)\left(\frac{k}{n}\right)^{\gamma} \int_{0}^{1} t^{\gamma}\left[\mathbb{V}_{n}^{-\gamma}\left(\frac{k}{n} t\right)-\left(\frac{k}{n} t\right)^{-\gamma}\right] d t,
\end{gathered}
$$

and

$$
T_{n 4}=\widehat{\gamma}_{k}-\gamma
$$

Next we show that $T_{n i}, i=1,2,3$ are asymptotically Gaussian rv's while $S_{n} \stackrel{p}{\rightarrow} \gamma+1$. First we consider the second term $T_{n 2}$. Once again, in view of the mean value theorem,

$$
\mathbb{V}_{n}^{-\gamma}\left(\frac{k}{n} t\right)-\left(\frac{k}{n} t\right)^{-\gamma}=-\gamma\left[\zeta_{n, k}(t)\right]^{-\gamma-1}\left(\mathbb{V}_{n}\left(\frac{k}{n} t\right)-\frac{k}{n} t\right)
$$

where $\zeta_{n, k}(t)$ is a sequence of rv's belongs to the open interval with endpoints $\mathbb{V}_{n}(k t / n)$ and $k t / n$. Then

$$
T_{n 2}=-\left(\widehat{\gamma}_{k}+1\right)\left(\frac{k}{n}\right)^{\gamma} \int_{0}^{1} t^{\widehat{\gamma}_{k}}\left(\frac{k}{n} t\right)^{-\gamma-1}\left[\mathbb{V}_{n}\left(\frac{k}{n} t\right)-\left(\frac{k}{n} t\right)\right] d t+R_{n}^{(2)},
$$

where

$R_{n}^{(2)}:=-\left(\widehat{\gamma}_{k}+1\right)\left(\frac{k}{n}\right)^{\gamma} \int_{0}^{1} t^{\widehat{\gamma}_{k}}\left[\left(\frac{n \zeta_{n, k}(t)}{k t}\right)^{-\gamma-1}-1\right]\left(\frac{k}{n} t\right)^{-\gamma-1}\left[\mathbb{V}_{n}\left(\frac{k}{n} t\right)-\left(\frac{k}{n} t\right)\right] d t$

By similar arguments as used below, we may easily show that $\sqrt{k} R_{n}^{(2)} \stackrel{P}{\rightarrow} 0$, therefore the details are omitted. By making use of the uniform empirical quantile process $\beta_{n}(\cdot)$, and by using the fact that $\widehat{\gamma}_{k} \stackrel{p}{\rightarrow} \gamma$, it becomes clear that

$$
\sqrt{k} T_{n 2}=\left(1+o_{p}(1)\right)(\gamma+1) \sqrt{\frac{k}{n}} \int_{0}^{1} t^{-1} \beta_{n}\left(\frac{k}{n} t\right) d t .
$$

By using similar arguments, we also show that

$$
\sqrt{k} T_{n 1}=-\left(1+o_{p}(1)\right) \gamma \sqrt{n / k} \beta_{n}\left(\frac{k+1}{n}\right) .
$$

Elementary computations give

$$
\sqrt{k} T_{n 3}=-\sqrt{k} \frac{\widehat{\gamma}_{k}-\gamma}{\widehat{\gamma}_{k}-\gamma+1} .
$$

Since $\widehat{\gamma}_{k} \stackrel{p}{\rightarrow} \gamma$, then $\sqrt{k} T_{n 3}=-\left(1+o_{p}(1)\right) \sqrt{k}\left(\widehat{\gamma}_{k}-\gamma\right)$. In view of the weak approximation (14), we get

$$
\begin{aligned}
& \sqrt{k} T_{n 1}=-\gamma \sqrt{n / k} B_{n}(k / n)+o_{p}(1) \\
& \sqrt{k} T_{n 2}=(\gamma+1) \sqrt{n / k} \int_{0}^{1} t^{-1} B_{n}(k t / n) d t+o_{p}(1)
\end{aligned}
$$


and

$$
\sqrt{k} T_{n 3}=-\sqrt{n / k} \int_{0}^{1} B_{n}(k t / n) d K_{\gamma}^{(1)}(t)+\sqrt{n / k} B_{n}(k / n) \int_{0}^{1} t d K_{\gamma}^{(1)}(t)+o_{p}(1) .
$$

It is easy to check that $S_{n}=T_{n 2}+T_{n 3}+\gamma^{-1}+1$. Since $\sqrt{k} T_{n i}, i=1,2,3$ are asymptotically Gaussian rv's, then $T_{n i} \stackrel{p}{\rightarrow} 0$. It follows that $S_{n} \stackrel{p}{\rightarrow} \gamma^{-1}+1$. Finally

$$
\begin{aligned}
\sqrt{k}\left(\frac{\widehat{a}_{n, k}}{a_{n, k}}-1\right) & =(\gamma+1) \sqrt{n / k} \int_{0}^{1} t^{-1} B_{n}(k t / n) d t-(\gamma+1) \sqrt{n / k} B_{n}(k / n) \\
& +\sqrt{n / k} \int_{0}^{1} B_{n}(k t / n) d K_{\gamma}^{(1)}(t)-\sqrt{n / k} B_{n}(k / n) \int_{0}^{1} t d K_{\gamma}^{(1)}(t)+o_{p}(1) .
\end{aligned}
$$

This may be rewritten into the simpler form

$$
\sqrt{k}\left(\frac{\widehat{a}_{n, k}}{a_{n, k}}-1\right)=\sqrt{\frac{n}{k}} \int_{0}^{1} B_{n}\left(\frac{k}{n} t\right) d K_{\gamma}^{(2)}(t)-\sqrt{\frac{n}{k}} B_{n}\left(\frac{k}{n}\right) \int_{0}^{1} t d K_{\gamma}^{(2)}(t)+o_{p}(1)
$$

where $K_{\gamma}^{(2)}(t):=(\gamma+1) \log t+a_{\gamma}^{-1} K_{\gamma}^{(1)}(t)$. For the second term of right hand-side of previous equation (15), we have first from the previous equation $\widehat{a}_{n, k} / a_{n, k}=1+o_{p}(1)$, and

$$
\sqrt{k}\left(\frac{\widetilde{a}_{n, k}}{a_{n, k}}-1\right)=\gamma \sqrt{n / k} B_{n}\left(\frac{k}{n}\right)+o_{p}(1)
$$

This latter compltetes the proof of Theorem 1.

\subsection{Proof of Corollary 1}

The proof is achived through straightforward and elementary computations. Therefore, it is omitted.

\subsection{Proof of Theorem 2}

Let us write

$$
\Psi\left[\widetilde{Q}_{n} ; g\right]-\Psi[Q ; g]=\Delta_{n 1}+\Delta_{n 2}
$$

where

$$
\Delta_{n 1}:=g(k / n) X_{n-k, n}+g(k / n) \frac{\rho \widehat{a}_{n, k}}{1-\rho \widehat{\gamma}_{k}}-\int_{0}^{k / n} Q(1-s) d g(s)
$$

and

$$
\Delta_{n 2}:=\sum_{i=k+1}^{n} a_{i, n}(g) X_{n-i+1, n}-\int_{k / n}^{1} Q(1-s) d g(s)
$$


It is easy to verify that

$$
\begin{aligned}
\frac{\sqrt{n} \Delta_{n 1}}{(k / n)^{-1 / 2} g(k / n) a_{n, k}} & =\gamma^{-1} \sqrt{k}\left(\frac{X_{n-k, n}}{Q(1-k / n)}-1\right)+\frac{\rho}{1-\rho \widehat{\gamma}_{k}} \sqrt{k}\left(\frac{\widehat{a}_{n, k}}{a_{n, k}}-1\right) \\
& +\frac{\rho^{2}}{\left(1-\rho \widehat{\gamma}_{k}\right)(1-\rho \gamma)} \sqrt{k}\left(\widehat{\gamma}_{k}-\gamma\right) \\
& +\sqrt{k}\left(\frac{1}{\gamma(1-\rho \gamma)}-\int_{0}^{k / n} \frac{Q(1-s)}{g(k / n) a_{n, k}} d g(s)\right) \\
= & \sum_{i=1}^{4} \Delta_{n 1}^{(i)} .
\end{aligned}
$$

In view of Theorem 1, we write

$\Delta_{n 1}^{(1)} \approx \sqrt{n / k} B_{n}\left(\frac{k}{n}\right)$

$\Delta_{n 1}^{(2)} \approx \frac{\rho}{1-\rho \gamma}\left\{\sqrt{\frac{n}{k}} B_{n}\left(\frac{k}{n}\right) \int_{0}^{1} t d K_{\gamma}^{(2)}(t)-\sqrt{\frac{n}{k}} \int_{0}^{1} B_{n}\left(\frac{k}{n} t\right) d K_{\gamma}^{(2)}(t)+\gamma \sqrt{\frac{n}{k}} B_{n}\left(\frac{k}{n}\right)\right\}$,

$\Delta_{n 1}^{(3)} \approx \frac{\rho^{2}}{(1-\rho \gamma)^{2}}\left\{\sqrt{\frac{n}{k}} B_{n}\left(\frac{k}{n}\right) \int_{0}^{1} t d K_{\gamma}^{(1)}(t)-\sqrt{\frac{n}{k}} \int_{0}^{1} B_{n}\left(\frac{k}{n} t\right) d K_{\gamma}^{(1)}(t)\right\}$.

Therefore

$$
\begin{array}{r}
\frac{\sqrt{n} \Delta_{n 1}}{(k / n)^{-1 / 2} g(k / n) a_{n, k}} \approx \sqrt{\frac{n}{k}} B_{n}\left(\frac{k}{n}\right) \int_{0}^{1} t d K_{\gamma}^{(3)}(t)-\sqrt{\frac{n}{k}} \int_{0}^{1} B_{n}\left(\frac{k}{n} t\right) d K_{\gamma}^{(3)}(t) \\
+\frac{1}{1-\rho \gamma} \sqrt{\frac{n}{k}} B_{n}\left(\frac{k}{n}\right)
\end{array}
$$

where

$$
K_{\gamma}^{(3)}(t)=\frac{\rho^{2}}{(1-\rho \gamma)^{2}} K_{\gamma}^{(1)}(t)+\frac{\rho}{1-\rho \gamma} K_{\gamma}^{(2)}(t)
$$

It was shown in Necir and Meraghni (2009), by using the second order condition (8), that

$$
\frac{\sqrt{n} \Delta_{n 2}}{(k / n)^{-1 / 2} g(k / n) a_{n, k}} \approx-\frac{\int_{0}^{1-k / n} g^{\prime}(1-t) B_{n}(t) d Q(t)}{(k / n)^{-1 / 2} g(k / n) a_{n, k}},
$$

and $\Delta_{n 1}^{(4)}$ tends to zero as $n \rightarrow \infty$. This achieves the proof of Theorem 2 .

\subsection{Proof of Corollary 2}

The proof is also achived through straightforward and elementary computations. Therefore, the details that can be found in Necir and Meraghni (2009) are omitted here . 
B. Brahimi, F. Meddi and A. Necir, Journal Afrika Statistika, Vol. 7, 2012, pages 474-490.

Bias-corrected estimation in distortion risk premiums for heavy-tailed losses.

\section{Conclusion}

In this paper we proposed a new estimator for the distortion risk premium when losses follow heavy-tailed distributions and established its asymptotic normality. This estimator improves, in term of bias, that of Necir and Meraghni (2009). The computation of such estimator depends on the choice of optimal number of upper extremes $k$ used in the tail index estimate. Such problem may be solved by using a heuristic method of Reiss and Thomas dicussed in Neves and Fraga Alves (2004). The software programs of this methodology are incorporated in the "Xtremes" package accompanying the book of Reiss and Thomas (2007).

Acknowledgements. The authors are greatly indebted to the referees for their valuable comments and suggestions.

\section{References}

Artzner, P., Delbaen, F., Eber, J. M. and Heath, D., 1999. Coherent measures of risk. Math. Finance. 9(3), 203-228.

Beirlant, J., Matthys, G., Diercks, G., 2001. Heavy-tailed distributions and rating. Astin Bull. 31(1), 37-58.

Beirlant, J., Diercks, G., Guillou, A. and Stărică, C., 2002. On exponential representations of log-spacings of extreme order statistics. Extremes. 5(2), 157-180.

Beirlant, J., Figueiredo, F., Gomes, M.I. and Vandewalle, B., 2008. Improved reduced-bias tail index and quantile estimators. J. Statist. Plann. Inference. 138(6), 1851-1870.

Brahimi, B., Meraghni, D., Necir, A. and Zitikis, R., 2011. Estimating the distortion parameter of the proportional hazard premium for heavy-tailed losses. Insurance Math. Econom. 49, 325-334.

Brazauskas, V., Jones, B. L.; Puri, M.L. and Zitikis, R., 2008. Estimating conditional tail expectation with actuarial applications in view. Journal of Statistical Planning and Inference. 138, 3590-3604.

Caeiro, F., Figueiredo, F. and Gomes, M.I., 2004. Bias reduction of a tail index estimator through an external estimation of the second order parameter. Statistics. 38(6), 497-510.

Caeiro, F., Gomes, M.I. and Rodrigues, L.H., 2009. Reduced-bias tail index estimators under a third-order framework. Comm. Statist. Theory Methods. 38(6-7), 1019-1040.

Centeno, M.L. and Andrade e Silva, J., 2005. Applying the proportional hazard premium calculation principle. Astin Bulletin. 35, 409-425.

Csörgő, M., Csörgő, S., Horváth, L. and Mason, D.M., 1986. Weighted empirical and quantile processes. Ann. Probab. 14(1), 31-85.

Denuit, M., Dhaene, J., Goovaerts, M.J. and Kaas, R., 2005. Actuarial Theory for Dependent Risk: Measures, Orders and Models. Wiley, New York.

Feureverger, A. and Hall, P., 1999. Estimating a tail exponent by modelling departure from a Pareto distribution. Ann. Statist. 27(2), 760-781.

Furman, E. and Zitikis, R., 2008a. Weighted premium calculation principles. Insurance Math. Econom. 42, 459-465.

Furman, E. and Zitikis, R., 2008b. Weighted risk capital allocations. Insurance Math. Econom. 43, 263-269.

Gomes, M.I. and Martins, M.J., 2002. "Asymptotically unbiased" estimators of the tail index based on external estimation of the second order parameter. Extremes. 5(1), 5-31. 
Gomes, M.I. and Martins, M.J., 2004. Bias reduction and explicit semi-parametric estimation of the tail index. J. Statist. Plann. Inference. 124(2), 361-378.

Gomes, M.I. and Figueiredo, F., 2006. Bias reduction in risk modelling: semi-parametric quantile estimation. Test. 15(2), 375-396.

Gomes, M.I. and Pestana, D., 2007. A sturdy reduced-bias extreme quantile (VaR) estimator. J. Amer. Statist. Assoc. 102(477), 280-292.

Greselin, F., Puri, M.L. and Zitikis, R., 2009. L-functions, processes, and statistics in measuring economic inequality and actuarial risks. Statistics and Its Interface. 2, 227-245.

de Haan, L. and Ferreira, A., 2006. Extreme Values Theory: An introduction. Springer.

Hill, B.M., 1975. A simple general approach to inference about the tail of a distribution. Ann. Statist. 3(5), 1163-1174.

Jones, B.L. and Zitikis, R., 2003. Empirical estimation of risk measures and related quantities. North American Actuarial Journal. 17, 44-54.

Jones, B.L. and Zitikis, R., 2007. Risk measures, distortion parameters, and their empirical estimation. Insurance Math. Econom. 41, 279-297.

Madan, D. B. and Schoutens, W., 2010. Conic financial markets and corporate finance. http://papers.ssrn.com/sol3/papers.cfm?abstract_id=1547022.

Mason, D., 1982. Laws of large numbers for sums of extreme values. Ann. Probab. 10(3), 754-764.

Matthys, G. and Beirlant, J., 2003. Estimating the extreme value index and high quantiles with exponential regression models. Statistica Sinica. 13, 853-880.

Matthys, G., Delafosse, E., Guillou, A. and Beirlant, J., 2004. Estimating catastrophic quantile levels for heavy-tailed distributions. Insurance Math. Econom. 34(3), 517-537.

Necir, A. and Meraghni, D., 2009. Empirical estimation of the proportional hazard premium for heavy-tailed claim amounts. Insurance Math. Econom. 45, no. 1, 49-58.

Necir, A. and Meraghni, D., 2010. Estimating L-functionals for heavy-tailed distributions and applications. Journal of Probability and Statistics 2010, ID 707146.

Neves, C. and Fraga Alves, M.I., 2004. Reiss and Thomas' automatic selection of the number of extremes. Comput. Statist. Data Anal. 47(4), 689-704.

Peng, L. and Qi, Y., 2004. Estimating the first- and second-order parameters of a heavytailed distribution. Aust. N. Z. J. Stat. 46(2), 305-312.

Peng, L., Qi, Y., Wang, R. and Yang, J., 2012. Jackknife empirical likelihood method for some risk measures and related quantities. Insurance Math. Econom. 51(1), 142-150.

Reiss, R.-D. and Thomas, M., 2007. Statistical Analysis of Extreme Values with Applications to Insurance, Finance, Hydrology and Other Fields, 3rd ed. Birkhäuser Verlag, Basel, Boston, Berlin.

Rolski, T., Schimidli, H., Schimd, V. and Teugels, J.L., 1999. Stochastic Processes for Insurance and Finance. John Wiley and Sons, New York.

Wang, S.S., 1996. Premium calculation by transforming the layer premium density. Astin Bulletin. 26, 71-92.

Weissman, I., 1978. Estimation of parameters and large quantiles based on the $k$ largest observations. J. Amer. Statist. Assoc. 73(364), 812-815.

Wirch, J.L. and Hardy, M.R., 1999. A synthesis of risk measures for capital adequacy. Insurance Math. Econom. 25, 337-347. 